\title{
Design and Modelling of a Novel Combustion Heat Exchanger for Household Heating
}

\author{
Bijan Yadollahi, Matthew Ritchie, Nader Karimi, Manosh C. Paul \\ Systems, Power and Energy Research Division, School of Engineering, University of Glasgow \\ G12 8QQ, Glasgow, UK \\ Bijan.Yadollahi@gla.ac.uk; 2078371R@student.gla.ac.uk ; Nader.Karimi@gla.ac.uk; Manosh.Paul@gla.ac.uk
}

\begin{abstract}
The present study is focused on the design and modelling of a novel Combustion Heat Exchanger (CHE), used for heating and hot water supplies in residential buildings. System design includes a combination of an efficient porous burner and heat exchangers. Combined with an Organic Rankine Cycle (ORC) and a Heat Pump (HP), it is meant to deliver higher energy efficiency as well as reduced greenhouse gas emissions. A numerical model has been developed in STAR-CCM+ to evaluate the design. Furthermore, system level heat transfer calculations were acquired to assist with the design process. A step by step approach was undertaken to investigate physical and chemical phenomena in the system. System dimensions, exchanger location and geometry, air/fuel ratio, porous media models, radiation and combustion were investigated along with different exchanger geometries. A novel spiral heat exchanger was introduced in addition to the common coil designs to exhibit both convection and radiation heat transfers. The results indicated that the exhibition of spiral heat exchanger would result in significantly enhanced heat transfer. Overall heat transfer coefficients of 4-5 times higher in comparison to coils could be expected for spiral exchangers. It was shown that radiation heat transfer accounts for a prominent share in the total heat transfer. Furthermore, the CHE could operate at a wide range of lean air/fuel ratios, enabling further decrease in greenhouse gas emissions. As the last part of the study, further investigations on the regular coil exchangers indicated that these exchangers could still be used with the design, but heat transfer enhancement is required to reduce the dimensions. Such enhancements were tested through shell geometry designs with improved results. Overall, the system shows a promising solution for further reduction of $\mathrm{CO}_{2}$ emissions while improving thermal efficiency.
\end{abstract}

Keywords: Combustion Heat Exchanger, Household Heating, Heat Transfer, Greenhouse gas emissions, Porous Burner, Computational Fluid Dynamics, STAR-CCM+, LTNE.

\section{Introduction}

Heat generation accounts for nearly half of the energy consumption and about a third of the carbon emissions in the UK [1]. Nearly eighty percent of the generated heat in the UK is used in household applications and other buildings [1]. Similar trends could be found in another countries, especially those with cold climates. The main energy sources for these appliances are fossil fuels. Therefore, even a small improvement in the performance of such systems, multiplied by the number of units, would result in a significant decrease in greenhouse gas emissions. Given the UK ambitious target to cut at least $80 \%$ of its greenhouse gas emissions by 2050 relative to 1990 levels [2], the significance of such a low carbon emitting system is even more pronounced.

Currently, natural gas is used as the main fuel supply of boilers generating heat for domestic and commercial sectors. Early versions of conventional boilers exhibited efficiencies of 50-65\% [3]. This has improved to 70-80\% due to different advances in boiler design [4]. Although conventional boilers are fairly efficient, there are still advances to be made to cut down the emissions from the use of these appliances.

Further development in boiler designs resulted in introduction of condensing boilers. This relatively new type has taken over, in both commercial and domestic water heating sectors, from the conventional boiler. In the condensing boiler, the exit gas temperature is reduced by the use of a second heat exchanger. In these appliances, the water vapour would condense and its latent heat is also used in heat transfer process. This gives the condensing boilers a much higher thermal efficiency when compared to the conventional boilers. Therefore, the condensing boilers produce fewer pollutant amounts in comparison with conventional types for a specific heat capacity. 
It has been indicated in Comakli [5] and Che et al. [6] studies that condensing boilers efficiency shows and increase of about $10 \%$ in comparison to conventional ones. Similar figures have been reported in Ref. [7]. The thermal efficiency of a condensing boiler is around 90\% [8]. This higher efficiency would translate to lower overall greenhouse gases and emissions. According to $\mathrm{Qu}$ et al. [4], the use of condensing boilers could reduce the $\mathrm{CO}_{2}$ emissions by $4 \%$.

Despite significant improvements in efficiency, condensing boilers are simply not enough to achieve future emission targets. There is a need in yet another level of efficiency improvement through more advanced concepts. Efficient combustion of lean air/fuel mixtures, enhanced heat transfer and waste heat recovery are key factors in further improving the performance of boilers.

All these factors could be integrated through a novel design to achieve maximum energy conversion efficiency. The proposed design combines a regular Organic Rankine Cycle (ORC) with a Heat Pump (HP) along with waste heat recovery. Utilizing this design, the total heat transfer to the working fluids (e.g. water) is even more than the heat generated by fuel combustion. More details about such a design can be found in [9]. At the heart of such a design lies a Combustion Heat Exchanger (CHE), which energizes the whole system.

The CHE is composed of an efficient burner and two heat exchangers, i.e. the ORC working fluid evaporator and post water heater. There is a need for high performance, low carbon emitting heat generation source as burner part. Flexibility of fuel (ranging from conventional fossil fuels to syngas and biogas), very high modulation ratios, stable operation even at very lean air/fuel mixtures and low emissions make porous burners a promising option for these heating systems.

Desirable combustion characteristics has led to extensive use of porous burners in different industry sectors of the industry [10,11].Among which applications of these burners for household applications such as cooking have also been reported [10]. Kamal and Mohamad have reported some novel and innovative burner designs for different applications [12]. Interesting characteristics of porous burners which make them a promising option for this application include [11,13]:

- an increased size of the reaction zone, since a more uniform temperature distribution,

- increased combustion stability,

- high potential to reduce emissions.

Application of porous burner along with heat exchanger for household applications has also been reported in a number of studies [14,15]. Durst et al. [14] suggested two different designs of axial and radial to achieve both performance and compactness. A combination of porous burner with solar heating has also been investigated [15]. Due to the complexities in the flow and combustion, design of these concepts has heavily relied on a thorough investigation of the flow field.

Present study aims at a more detailed description on design and optimization of the CHE. The design concept has been briefly described at first, and more details about each part have been presented. The design process included system level heat transfer calculations along with detailed investigations of the flow field. Heat transfer calculations were performed using an extended spreadsheet to evaluate the designs in terms of heat balance. Design variants could be checked much faster using this approach. Furthermore, a Computational Fluid Dynamics (CFD) model has been utilised to investigate the flow field in the system. The CFD tool also enabled further optimisation of the design.

The flow field in the CHE involves complicated interactions between the flow, turbulence, all modes of heat transfer, the porous medium and chemistry. Proper refinement in the numerical model was absolutely necessary in order to reflect all these interactions accurately. Therefore, a step-by-step approach was undertaken to start from a basic model and add details of models as well as geometry gradually. Rigorous validations were performed to ensure validity of results in each step.

In the design of heat exchangers, ability to deliver required heat transfer while keeping the dimensions in a reasonable level is of a primary importance. Therefore, a key part of the investigation included design and optimisation of the heat exchangers. Different exchanger designs were tested in the CFD model. Particularly, a novel spiral design was introduced to exhibit both convective and radiative heat transfers, as the latter is prominent in the porous burners. The last part of the study includes an investigation of heat transfer enhancement in case it is not adequate for the exchanger design.

\section{Model Characterization}

The CHE has been a part of a larger system. Therefore, the design would have interfaces to other system components. Main interface data include required heat loads in heat exchangers, flow conditions at inlet and outlet of each exchanger, minimum temperature of flue gases exiting the $\mathrm{CHE}$ and thermo-physical properties of the working fluids. Furthermore, it was intended to use natural gas as the main fuel. These interface data have initially been determined via thermodynamic calculations for the whole system, as reported in [9]. The same set of interface data have been used as boundary conditions for the CHE design. The design itself included iterations between system level heat 
transfer calculations and detailed numerical models. Details of model evolutions have been presented in following sections.

\subsection{Design Concept}

$\mathrm{CHE}$ is the main driving source of the whole system. It should provide required heat load by working cycles to operate them. The main tasks of the CHE are:

- providing the required heat for the ORC cycle evaporator,

- providing the required heat for the post water heater,

- delivering hot enough flue gases for later mixing with cold air.

Therefore, the CHE should at least include a burner and two heat exchangers. A concept design has been developed based on the requirements for a $20 \mathrm{~kW}$ CHE. The proposed design has been shown in Fig. 1. Different parts of the system have been designated in the figure. It consists of two heat exchangers, porous zones, an igniter and a casing.

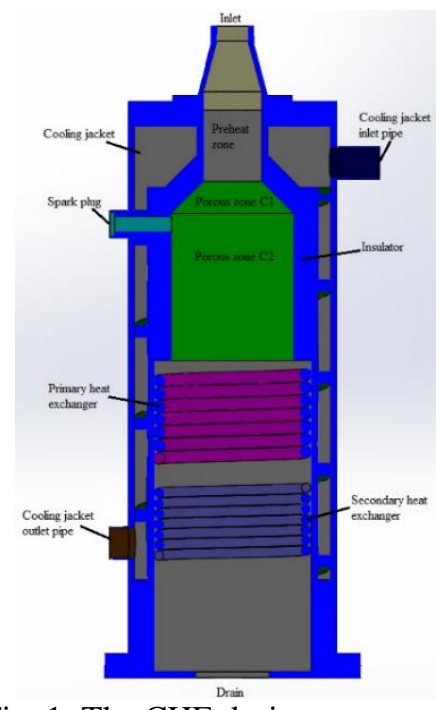

Fig. 1: The CHE design concept.

Three porous parts with different properties have been used in the design. The first part is in the preheat zone, which is responsible for both pre-heating the mixture of fuel/air and preventing flame flashback. Combustion occurs in zones $\mathrm{C} 1$ and $\mathrm{C} 2$ (see Fig. 1), which are other porous zones in the system. Combustion stability and operating considerations necessitates these two sections to be of different porosities as well. A layer of insulator separates the combustion zone from the outer shells for safety reasons. The porous zones were selected from ceramic foams. Among available options of $\mathrm{Al}_{2} \mathrm{O}_{3}, \mathrm{ZrO}_{2}$ and $\mathrm{SiC}$, the preheat zone is made from alumina with $20 \mathrm{PPI}$ porosity. Zone $\mathrm{C} 1$ and $\mathrm{C} 2$ were made of $\mathrm{SiC}$ with porosities of 20 PPI and 10 PPI respectively. These selections were made based on the initial investigations on the characteristics of these porous foams.

An igniter is used to start combustion in the porous zones. The outer casings have been made in two layers, with a cooling jacket in between. Cold water circulates through the channels and cools the system down to safe temperatures (about $40^{\circ} \mathrm{C}$ ). This ensures that the temperature of outer shell would be much lower than the combustion temperature.

In the proposed design, the premixed mixture of natural gas and air enters through the inlet. This mixture is heated up in the preheat zone, and then enters the combustion zone. Hot combustion products move towards the heat exchangers, where heat transfer occurs and the gas temperature drops significantly. Same process is undergone in the second heat exchanger, and flue gases are directed towards the outlet drain section. The system would be installed straight to help drain condensed water by the use of gravity.

Radiation takes a significant share of heat transfer in porous burners. Therefore, a novel design of spiral heat exchangers was introduced to take advantage of this heat transfer as well. This exchanger shows a large view factor because of the diskshaped surfaces. Since the disk is located directly opposite to the burner outlet, the exchanger tubes lie in the active flow zone, and even the convective heat transfer could be enhanced with this design. Regular coil heat exchanger designs were 
also tried to investigate their performance. This design is the base for further analysis using computational tools. More details on the design could be found in [16,17].

\subsection{Numerical Model Development}

A numerical model of the system has been developed in STAR-CCM+. The first step towards developing the numerical model is extracting the so called "empty volume" out of the CHE model. This is the empty space in the model enclosed by all boundaries. An adequately refined computational grid should be created in this geometry. A separate part of investigation has been dedicated to determine cell size.

Exchanger location and geometry, air/fuel ratio, porous media models, radiation and combustion were investigated. Detailed geometry along with multiple physical and chemical phenomena in the model necessitates large number of cells, and consequently, long runtimes. Therefore, it was decided to start from a basic model, and add details step by step. A series of "simplified" geometries were created in the process. The need to perform detailed modelling reduced considerably using this method. Furthermore, the validity of results was checked at each step using benchmark cases.

Mass flow and total temperature were set as inlet boundary conditions. These quantities were set according to the desired relative air to fuel ratio $(\lambda)$. It is assumed that natural gas is completely made of Methane. Standard air conditions were applied at outlet. Casing wall thermal specifications were set as constant temperature. Due to the use of insulator around the combustion zone, the walls in that region were set as adiabatic. Walls of the heat exchangers were treated as constant average temperature. In cases of reactive flow, fuel and air mass fractions were set at inlet. These values were also determined based on the desired lambda. The objective is to operate the burner at lean lambda to increase the efficiency and reduce emissions. Therefore, the lambda has been chosen to lie in lean range, i.e. greater than unity.

There are two ways to model a porous media, namely the Local Thermal Equilibrium (LTE) and Local Thermal NonEquilibrium (LTNE). The former assumes thermal equilibrium between fluid and solid phases of the porous media, while the latter does not consider such an equilibrium. In LTNE case, a separate energy equation is solved for each phase, giving more refined results. Both these models were investigated through the modelling, aiming at a more detailed understanding of the porous media. Where possible, the LTNE model has been used for more refinement.

Interaction between combustion and porous media on one side, and with the flow and turbulence on the other side requires use of a detailed chemistry rather than simplified models. Therefore, a reduced mechanism of 30 species and 184 reactions for Methane/air combustion was used [18].

The flow was assumed to be steady state, three dimensional and incompressible. The k- $\varepsilon$ turbulence model was used, and second order discretization was applied to all equations. The equations were solved using coupled solver. Solution iteration continued until residual levels reduced to $10^{-6}$ for energy equation (which was the slowest converging equation). More details about numerical model development could be found in Ref. [19].

\subsection{Grid Independency and Validation}

A part of the investigation was dedicated to determine the adequate cell size. Furthermore, it is necessary to ensure validity of the results, particularly that of porous media, radiation and combustion models. Therefore, three separate benchmark cases, including those of Yang and Vafai [20] on porous media, a classic radiation heat transfer from Ref. [21], and investigation of Moraga et al. [22] on combustion in porous media were selected to assess the numerical model.

A comparison of the non-dimensional temperature for LTE and LTNE cases has been shown in Fig. 2-a. The x-axis represents non-dimensional length across channel half length, where 0 and 1 denote the channel centreline and walls respectively. Good agreement between the two porous media models could be seen in the figure.

The radiation validations includes a hot plate at $1000 \mathrm{~K}$, underneath a curved absorber where the heat is transferred through the air, and other wall temperatures are $300 \mathrm{~K}$. A view of the temperature field in this case has been shown in Fig. 2-b. The total calculated radiation heat transfer in the present study is $81.4 \mathrm{~kW}$ compared to $77.1 \mathrm{~kW}$ in the analytical solution, showing a mere $5.75 \%$ error. 


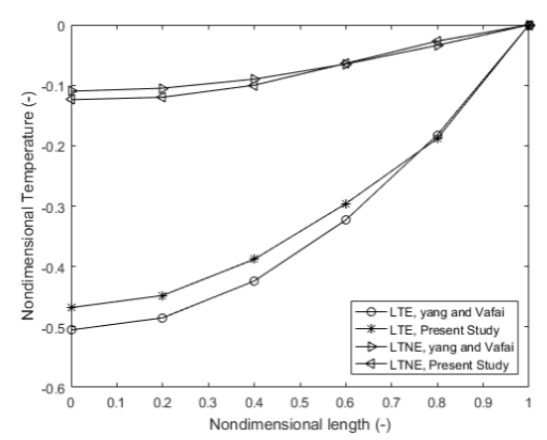

a

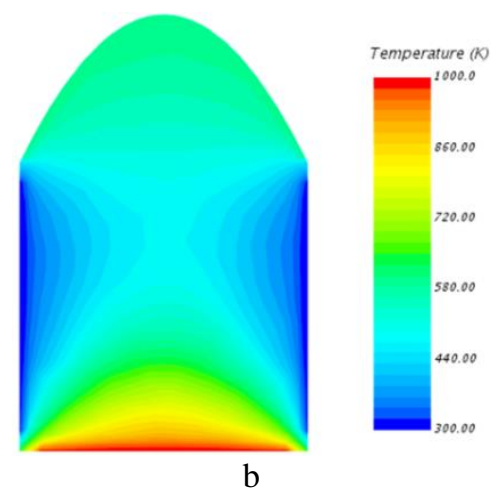

Fig. 2: Validation results, a: porous media, b: radiation.

The combustion validation case of Moraga et al. [22], combines the porous media and combustion. The effect of radiation has been accounted for through an average thermal conductivity. The geometry is a round channel filled with porous material, and Methane/air combustion has been investigated in different porosities and $\lambda$ values. A coarse grid of 800000 cells and a fine mesh with 1430000 cells were produced for this investigation, to ensure the proper grid size. A comparison between the results of combustion temperature has been presented in Table 1 .

Table 1: Comparison of results in combustion validation test case.

\begin{tabular}{|c|c|c|c|c|c|c|}
\hline \multirow{2}{*}{$\lambda(-)$} & \multirow{2}{*}{ Porosity (-) } & \multirow{2}{*}{ Inlet velocity $(\mathrm{m} / \mathrm{s})$} & \multicolumn{3}{|c|}{ Maximum gas Temperature $(\mathrm{K})$} & \multirow{2}{*}{ Maximum error (\%) } \\
\cline { 4 - 6 } & & & Moraga et al. & Coarse grid & Fine grid & \\
\hline 1.94 & 0.4 & 0.43 & 1640.45 & 1581.9 & 1593.7 & 3.6 \\
\hline 2.83 & 0.4 & 0.43 & 1474.57 & 1446.6 & 1453.2 & 1.9 \\
\hline 2.83 & 0.6 & 0.43 & 1437.38 & 1423.4 & 1427.5 & 0.098 \\
\hline
\end{tabular}

Comparison of the data in Table 1 shows excellent agreement between the current results and the benchmark case. Furthermore, the difference between the results in fine and coarse grids are not significant. This indicates the adequate cell size for the numerical model. Similar settings have been used in the model for main investigations. Overall, investigations performed in this section show the results of numerical model are valid.

\section{Results and Discussion}

The first objective was to determine the heat transfer coefficient over each spiral and coil designs, and feed these data to the overall heat transfer calculations. Due to the large number of design iterations, hot gas was used instead of combustion products, but the temperature and mass flow rate were kept the same as theoretical values with the same $\lambda$.

Heat transfer calculations showed the need for more than one disk to deliver the required heat transfer in spiral exchanger. Therefore, accurate positioning of the disks was of major importance to ensure proper heat transfer over all disks. Proper positioning of the first disk relative to the burner outlet was also vital for radiation heat transfer. A view of velocity and temperature contour plots for such an investigation has been shown in Fig. 3.

It could be seen in Fig. 3 that the first two disks create a wide wake region, affecting flow over other disk. The top two disks are connected and form a single heat exchanger. The second heat exchanger has been positioned optimally to lie outside the wake region for higher heat transfer coefficients. Investigations in different $\lambda$ indicated optimal location for the first and second disk at 3 and $6 \mathrm{~cm}$ downstream to burner outlet respectively. Optimal location for the secondary heat exchanger was at $18 \mathrm{~cm}$ downstream to the burner outlet. The heat transfer coefficient ranges near $70 \mathrm{~W} / \mathrm{m}^{2} \mathrm{~K}$. Radiation contributes to a significant share of this value (between 12-22\%). 


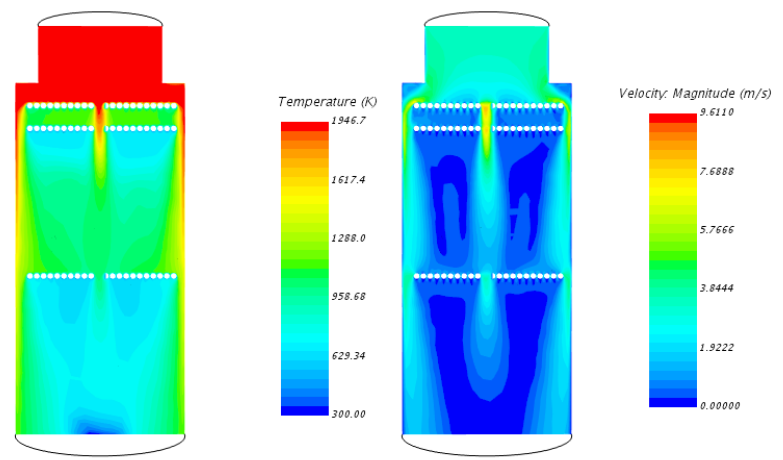

Fig. 3: Temperature and velocity fields for spiral design on a plane passing though axis, at $\lambda=1$.

Combustion investigations provided more accurate data for heat transfer calculations. These investigations were performed on the complete model with all three porous zones included, using LTNE model. Some snapshots of the flow field have been depicted in Fig. 4. Uniform distribution of the high temperature zone and species could be observed in the figure. These investigations indicated that the $\lambda$ values over 2.0 could also be used with desirable flow field.

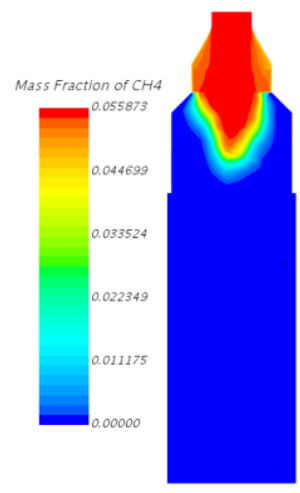

a

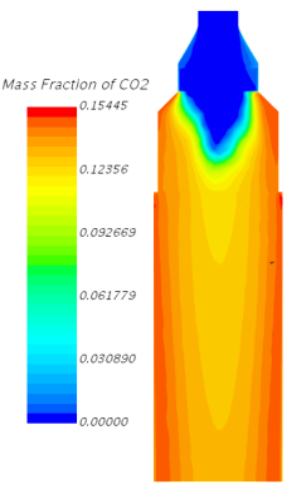

b

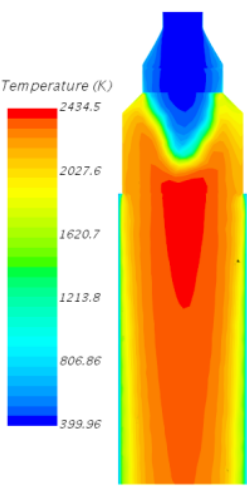

c

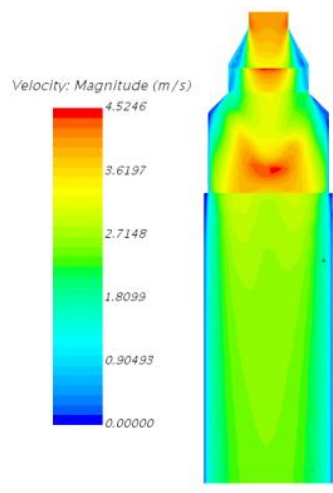

d

Fig. 4: Combustion at $\lambda=1.0$ and $\mathrm{T}_{\text {Inlet }}=450 \mathrm{~K}$, a: mass fraction of $\mathrm{CH}_{4}$, b: mass fraction of $\mathrm{CO}_{2}$, c: temperature, d: velocity.

Coil exchangers could barely benefit from the radiative heat transfer. Therefore, adequate convective heat transfer coefficient is central to their performance. In this application, the original coils were designed with maximum available base circle diameter $(14 \mathrm{~cm})$ and higher tube diameters $(16 \mathrm{~mm})$ to reduce their number of revolutions. However, this proved problematic as the heat transfer coefficients went very low $\left(\sim 9 \mathrm{~W} / \mathrm{m}^{2} \mathrm{~K}\right)$. Therefore, the optimal base circle design was investigated, resulting in the modified base diameter of $10 \mathrm{~cm}$ with higher heat transfer coefficients of roughly $13 \mathrm{~W} / \mathrm{m}^{2} \mathrm{~K}$. A comparison of the results in this case has been shown in Fig. 5. It is clear in the figure that the modified coil has lied in the region with higher velocities. While this is a significant improvement to the original design, it resulted in the need for longer coils, with even poorer heat transfer coefficient $\left(7 \mathrm{~W} / \mathrm{m}^{2} \mathrm{~K}\right)$. Heat transfer enhancement using different insert geometries was also investigated (Fig. 6). Radiation is also included in these investigations.

The flow is originally asymmetric because of the presence of tube connections to the outside shell. Addition of the cylinder inserts made this asymmetry even more pronounced, probably because of the separations and blockage effect. However, inserts with refined design (e.g. cone shape in Fig. 6-b), resulted directing the flow towards exchanger coil and hence increase in heat transfer coefficient by nearly $45 \%$. Using a cone removed the weak flow zone at the top, helped in distributing flow more evenly (as is obvious in Fig. 6), and helped increasing heat transfer coefficient in both heat exchangers. The best heat transfer coefficients in this case were near 11.5 for both heat exchangers. 

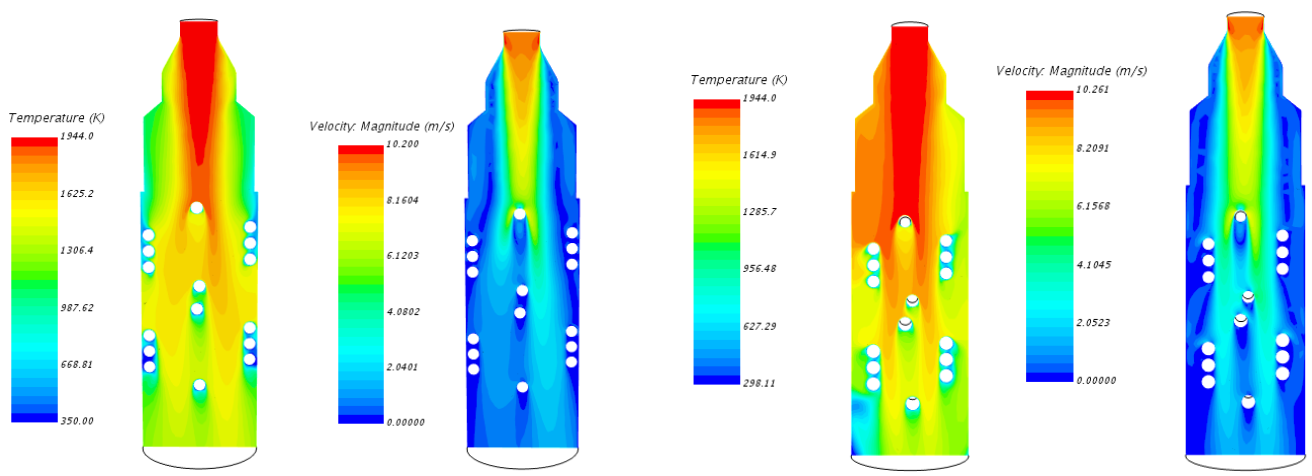

$\mathrm{b}$

Fig. 5: Velocity and temperature on a plane passing though axis at $\lambda=1$ for coil designs, a: initial design, b: modified design.
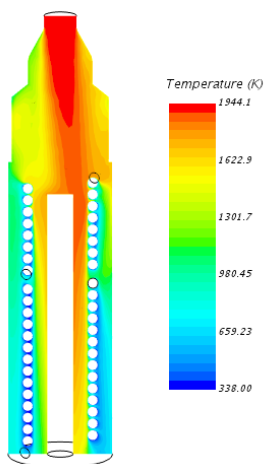

$8=8$

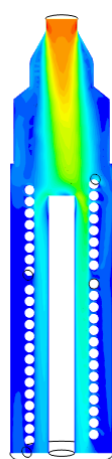

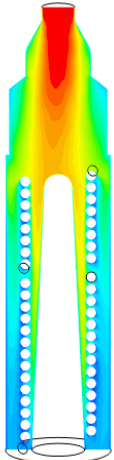
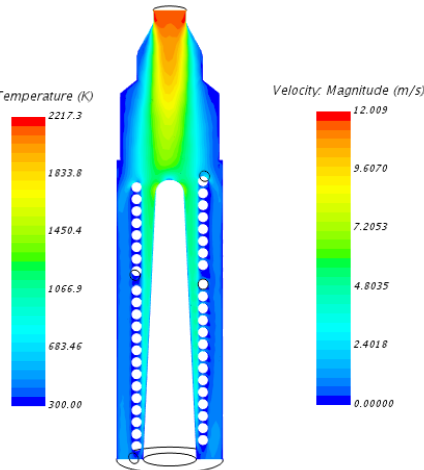

$\mathrm{b}$

Fig. 6: flow field comparison for two different inserts used for heat transfer enhancement, a: cylinder, b: cone.

\section{Conclusion}

Main concluding remarks from the present study have been summarized as follows.

- A novel CHE with higher thermal efficiency and lower emissions has been proposed for household applications.

- The design incorporates an efficient, lean burn porous burner along with novel, spiral heat exchanger designs.

- A numerical model has been developed in STAR-CCM+ to investigate the design and expedite it.

- Three validations test cases have been performed to ensure validity of results. Comparison indicates good agreement between the results of present study and benchmark data.

- Spiral exchangers show 4-5 times higher heat transfer coefficient in comparison to coil designs.

- The share of radiation in heat transfer coefficient of spiral exchangers varies between $12-22 \%$.

- Combustion investigations indicate $\lambda$ values of higher than 2.0 could be used.

- Original coil designs show weak heat transfer coefficient. Modification of base circle diameter helps in the increase, but results in longer coils with poor performance. Cone inserts with refined design could partially solve the problem.

\section{Acknowledgements}

Funding: Engineering and Physical Research Council, EPSRC, UK, project number EP/N020472/1 (Therma-Pump).

\section{References}

[1] L. Fabrice and F. Robertson, (2014), Pathways for Heat: Low Carbon Heat for Buildings. A report by Carbon Connect. [Online]. Available: http://www.igem.org.uk/media/343656/carbonconnect_pathwaysforheat_webcopy.pdf

[2] UK Government, (2009), The UK Low Carbon Transition Plan. [Online]. Available: http://www.carbonaction2050.com/files/documentattachment/UKLowCarbonTransitionPlan.pdf

[3] P. Basu, C. Kafa, and L. Jestin, Boilers and Burners: Design and Theory. Springer - Verlag, New York, 2000. 
[4] M. Qu, O. Abdelaziz, and H. Yin, "New configurations of a heat recovery absorption heat pump integrated with a natural gas boiler for boiler efficiency improvement," Energy Conv. Manag., vol. 87, pp. 175-184, 2014.

[5] K. Comakli, "Economic and environmental comparison of natural gas fired conventional and condensing combi boilers," J Energy Institute, vol. 81, pp. 242-246, 2008.

[6] D. Che, Y. Liu, and C. Gao, "Evaluation of retrofitting a conventional natural gas fired boiler into a condensing boiler," Energy Conversion and Management, vol. 45, no. 20, pp. 3251-3266, 2004.

[7] S. Lee, S.M. Kum, and C.E. Lee, "Performances of a heat exchanger and pilot boiler for the development of a condensing gas boiler," Energy, vol. 36, pp. 3945-3951, 2011.

[8] Q. Chen, K. Finney, H. Li, X. Zhang, J. Zhou, V. Sharifi, and J. Swithenbank, "Condensing boiler applications in the process industry," Applied Energy, vol. 89, no. 1, pp. 30-36, 2012.

[9] Y. Liang, M. Al-Tameemi and Z. Yu, "Investigation of a gas-fuelled water heater based on combined power and heat pump cycles," J. Applied Energy, vol. 212, pp. 1476-1488, 2018.

[10] M. A. Mujeebu, M. Z. Abdullah, M. Z. Abu Bakar, A. A. Mohamad, R. M. N. Muhad and M. K. Abdullah, "Combustion in porous media and its applications - A comprehensive survey," J. Envir. Manag., vol. 90, no. 8, pp. 2287-2312, 2009.

[11] D. Trimis and F. Durst, "Combustion in a Porous Medium - Advances and Applications," Combustion Sci. and Tech., vol. 121, pp. 153-168, 1996.

[12] M. M. Kamal and A. A. Mohamad, "Combustion in Porous Media," Proc. Inst. Mech. Eng. Part A: J. Power and Energy, vol. 220, no. 5, pp. 487-508, 2006.

[13] G. Brenner, K. Pickenäcker, O. Pickenäcker, D. Trimis, K. Wawrzinek, and T. Weber, "Numerical and Experimental Investigation of Matrix-Stabilized Methane/Air Combustion in Porous Inert Media," Combustion and Flame, vol. 123, pp. 201-213, 2000.

[14] F. Durst, D. Trimis, K. Pickenäcker, (1997), Compact Porous Medium Burner and heat Exchanger for Household Applications. Project report. [Online]. Available: https://cordis.europa.eu/docs/publications/5465/54656401-6_en.pdf

[15] F. Avdic, "Application of the Porous Medium Gas Combustion Technique to Household Heating Systems with Additional Energy Sources," Ph.D. dissertation, Dept. Tech., Universität Erlangen, Nürnberg, Germany, 2004.

[16] M. Hamilton, "Design and Manufacture of a Porous Medium Burner for Residential Heating," MSc. Dissertation, School of Engineering, University of Glasgow, Glasgow, UK, 2017.

[17] D. Niven, "Design and Analysis of a Novel Domestic Boiler," MEng. Dissertation, School of Engineering, University of Glasgow, Glasgow, UK, 2018.

[18] T. Lu and C. K. Law, "A Criterion Based on Computational Singular Perturbation for the Identification of Quasi Steady State Species: a Reduced Mechanism for Methane Oxidation with NO Chemistry," Combustion and Flame, vol. 154, no. 4, pp. 761-774, 2008.

[19] M. Ritchie, "Computational analysis of a new concept boiler in order to optimise the design and investigate the thermodynamic effects", MEng. Dissertation, School of Engineering, University of Glasgow, Glasgow, UK, 2018.

[20] K. Yang, and K. Vafai, "Analysis of temperature gradient bifurcation in porous media - An exact solution," Int. $J$ Heat Mass Tran., vol. 53, pp. 4316-4325, 2010.

[21] F. P. Incropera, D. P. DeWitt, T. L. Bergman, and A. S. Lavine, Fundamentals of Heat and Mass Transfer, 6th ed. John Wiley and Sons Ltd, 2010.

[22] N. O. Moraga, C. E. Rosas, V. I. Bubnovich, and N. A. Solari, "On predicting two-dimensional heat transfer in a cylindrical porous media combustor," Int. J Heat Mass Tran., vol. 51, pp. 302-311, 2008. 